\title{
Interface Sharpness in the Ising System
}

\author{
H. van Beijeren $\star$ \\ Institute for Theoretical Physics, Katholieke Universiteit Nijmegen, \\ Nijmegen, The Netherlands
}

Received July 22, 1974

\begin{abstract}
A simple proof is given for the existence of a sharp interface in threedimensional Ising systems, at least up to the critical temperature of the corresponding two-dimensional system.
\end{abstract}

\section{Introduction}

For the three-dimensional Ising model with nearest neighbour interactions on a simple cubic lattice Dobrushin has shown [1] that at low enough temperature there can exist a sharp interface between areas of opposite magnetization. A horizontal sharp interface is characterized by a vertical level $l$ and a positive constant $a$, independent of the size of the system, such that the expectation values of all spins above $l$ are $>a$ and those of spins below $l$ are $<-a$, or vice versa. The spin system can be forced into a state possessing such an interface by applying a positive magnetic field to all the boundary spins above the level $l$ and a negative magnetic field to all the boundary spins below $l$.

On the other hand for the two-dimensional square Ising model with nearest neighbour interactions Gallavotti has shown [2] that even at very low non-zero temperature no sharp interface exists. By lowtemperature series expansions Weeks, Gilmer, and Leamy [3] found strong evidence that the three-dimensional system has a "rougheningtemperature" $T_{R}$ above which the interface is no longer sharp. For this roughening temperature they find values of about 0.57 times the critical temperature $T_{c}$. This is somewhat larger than the critical temperature of the corresponding two-dimensional system, which is about half of the critical temperature of the three-dimensional system.

Here we give a very simple proof that the critical temperature of the two-dimensional system is a lower bound on the roughening temperature $T_{R}$. We use a variant of Percus' method of the "duplicate set of variables", which has recently been described by Lebowitz $[4]^{1}$. Moreover we will

* Present address: Institute for Fluid Dynamics and Applied Mathematics, University of Maryland, College Park, Maryland 20740, USA. 
discuss some simple generalizations of our result, as well as some consequences of the inequalities which were derived by Lebowitz in Ref. [4].

\section{The Main Result}

We consider a simple cubic lattice on a cube of $2 N+1$ horizontal layers numbered $-N,-N+1, \ldots, N$. The spins on the central layer, 0 , are numbered $\sigma_{m}, \sigma_{n} \ldots$, those on the layers $1, \ldots N$ are numbered $\sigma_{i}, \sigma_{j} \ldots$ and those on the layers $-1, \ldots-N$ are numbered $\sigma_{-i}, \sigma_{-j} \ldots$ This numbering is chosen so that the sites $i$ and $-i$ are each others mirror image with respect to the central layer. Furthermore we consider a twodimensional square lattice of $(2 N+1) \times(2 N+1)$ sites with spins numbered $\sigma_{m}^{\prime}, \sigma_{n}^{\prime} \ldots$. All the spins in both systems may assume the values \pm 1 only. The Hamiltonians of both systems are given as

$$
\begin{aligned}
& H(\boldsymbol{\sigma})=J\left\{\sum_{[i j]}\left(\sigma_{i} \sigma_{j}+\sigma_{-i} \sigma_{-j}\right)\right.\left.+\sum_{[m n]} \sigma_{m} \sigma_{n}+\sum_{[i m]} \sigma_{m}\left(\sigma_{i}+\sigma_{-i}\right)\right\} \\
&+\sum_{i} h_{i}\left(\sigma_{i}-\sigma_{-i}\right)+\sum_{m} H_{m} \sigma_{m}, \\
& H^{\prime}\left(\boldsymbol{\sigma}^{\prime}\right)=J \sum_{[m n]} \sigma_{m}^{\prime} \sigma_{n}^{\prime}+\sum_{m} H_{m} \sigma_{m}^{\prime} .
\end{aligned}
$$

The square brackets denote that the summations are restricted to pairs of nearest neighbours. All the $h_{i}$ and $H_{m}$ must be non-negative. We are especially interested in the case where $h_{i}$ and $H_{m}$ are $+\infty$ at the boundary sites and zero at all other sites. Then at low temperatures we expect an interface between the layers 0 and 1 .

In analogy with the method of Percus ${ }^{1}$ we change to the variables

$$
\begin{aligned}
s_{i} & =\frac{1}{2}\left(\sigma_{i}+\sigma_{-i}\right), & t_{i} & =\frac{1}{2}\left(\sigma_{i}-\sigma_{-i}\right), \\
s_{m} & =\frac{1}{2}\left(\sigma_{m}+\sigma_{m}^{\prime}\right), & t_{m} & =\frac{1}{2}\left(\sigma_{m}-\sigma_{m}^{\prime}\right),
\end{aligned}
$$

which may assume the values $-1,0,1$, with the constraint

$$
s_{i}= \pm 1 \Rightarrow t_{i}=0 \quad \text { and } \quad s_{i}=0 \Rightarrow t_{i}= \pm 1
$$

The sum of $H$ and $H^{\prime}$ can be expressed in these new variables as

$$
\begin{aligned}
H(\boldsymbol{\sigma})+H^{\prime}\left(\boldsymbol{\sigma}^{\prime}\right)=2 J\left\{\sum_{[i j]}\left(s_{i} s_{j}+t_{i} t_{j}\right)\right. & \left.+\sum_{[m n]}\left(s_{m} s_{n}+t_{m} t_{n}\right)+\sum_{[i m]}\left(s_{i} s_{m}+s_{i} t_{m}\right)\right\} \\
& +\sum_{i} 2 h_{i} t_{i}+\sum_{m} 2 H_{m} s_{m} .
\end{aligned}
$$

In the same way as in Ref. [4] the Griffiths, Kelly, and Sherman inequalities [5] can be used to obtain the result:

$$
\left\langle t_{m}\right\rangle \geqq 0,
$$

1 In contrast to Ref. [4] we do not combine two identical systems, but we combine one system partly with itself and partly with another system. 
where the brackets denote an expectation value with respect to the product measure of the canonical measures for $\boldsymbol{\sigma}$ and $\boldsymbol{\sigma}^{\prime}$ respectively. This expectation value may be considered as a canonical average over the $s$ and $t$ variables with the Hamiltonian (4) under the constraint (3). This average on its turn can be written as a weighted sum of partial averages in each of which half of the $s$ and $t$ variables are kept fixed at zero and the remaining ones assume the values \pm 1 . Each partial average by itself is a canonical average for an Ising system with ferromagnetic interactions, to which the GKS inequalities apply.

The implication of (5) is that the average magnetization of the central layer is larger than the average magnetization in a corresponding twodimensional Ising system with equal, positive, boundary fields.

It is interesting also to consider the system with $2 N$ layers and similar boundary conditions, where there is a symmetry between the upper and the lower half of the system. The $2 N$-layer system can be obtained in the following way: Start with a $2 N+1$-layer system with boundary fields $+\infty$ above the level -1 and $-\infty$ below the level 0 , and apply a field $+\infty$ to all spins in the layer $N-1$. The resulting system is antisymmetric with respect to the plane between the layers 0 and -1 . From the Fortuin, Kastelein and Ginibre inequalities [6] it follows that the average magnetization in the layer 0 is not decreased by applying a positive field to the spins in the layer $N-1$, hence this magnetization is still larger than the average magnetization in the corresponding two-dimensional Ising system. By symmetry the average magnetization in the layer -1 is exactly the opposite of that in the layer 0 . [Notice that this magnetization was even more strongly negative in the $(2 N+1)$-layer case, as a consequence of the FKG inequalities.] Hence we may conclude that under the given boundary conditions there is indeed a sharp interface between the layers 0 and -1 .

\section{Some Simple Generalizations}

Some straightforward extensions of the result obtained in Section 2 are listed below. In all these extensions (5) remains true if the central layer is compared with a two-dimensional Ising system of the same shape, with the same, ferromagnetic, coupling constants and with the same, nonnegative external fields.

1. The lattice need not be a cube. Both the central layer and the other layers may be of arbitrary shape, as long as the layers $n$ and $-n$ are each others mirror image with respect to the central layer.

2. The restriction to nearest neighbour couplings of equal strength may be loosened: First the strength of different nearest neighbour inter- 
actions may be different, provided the couplings between pairs $i, j$ and $-i,-j$ or $i, m$ and $-i, m$ remain equal. An interesting case is the so-called solid-on-solid model [3], frequently used in the theory of crystal growth, which has a coupling of finite strength $J$ between horizontal nearest neighbours and a coupling of infinite strength between vertical nearest neighbours. It follows that also in this model $T_{R}$ is not smaller than the critical temperature of the corresponding two-dimensional Ising model. This is consistent with results obtained in computer experiments by crystal growth theorists [7].

Furthermore it is allowed to add arbitrary ferromagnetic interactions which do not involve spins both above and below the central layer, provided again the symmetry between above and below is maintained. For instance it is allowed to add ferromagnetic couplings anywhere between next-nearest and next-next-nearest neighbour pairs. It is even allowed to add an interaction between symmetric pairs of spins in the layers 1 and -1 ; such interactions give rise to terms of type $s_{i}^{2}-t_{i}^{2}$ in the Hamiltonian (4), but these are allowed since they are constant in each partial average, where a given set of $s$ and $t$ variables is kept fixed to zero. It is not allowed however to add any other interactions which couple spins in vertical layers above and below the central layer, since this would introduce antiferromagnetic terms in the Hamiltonian (4).

\section{Additional Results}

In this section we consider the three-dimensional system by itself without combining it with a two-dimensional layer. The Hamiltonians for the $(2 N+1)$-layer case and the $2 N$-layer case respectively can be written as

$$
\begin{gathered}
H^{2 N+1}(\boldsymbol{\sigma})=2 J\left\{\sum_{[i j]}\left(s_{i} s_{j}+t_{i} t_{j}\right)+\frac{1}{2} \sum_{[m n]} \sigma_{m} \sigma_{n}+\sum_{[i m]} s_{i} \sigma_{m}\right\} \\
+\sum_{i}\left(2 h_{i} t_{i}+2 H_{i} s_{i}\right)+\sum_{m} H_{m} \sigma_{m} \\
H^{2 N}(\boldsymbol{\sigma})=2 J \sum_{[i j]}\left(s_{i} s_{j}+t_{i} t_{j}\right)+J \sum_{i \in\{1\}}\left(s_{i}^{2}-t_{i}^{2}\right)+\sum_{i}\left(2 h_{i} t_{i}+2 H_{i} s_{i}\right),
\end{gathered}
$$

where $\{1\}$ denotes the layer 1 and where we have allowed for nonnegative fields $H_{i}$ and $H_{m}$ acting upon $s_{i}$ and $\sigma_{m}$ respectively.

The following inequalities, derived by Lebowitz in Ref. [4] can immediately be shown to hold here as well: 


$$
\begin{gathered}
\text { Interface Sharpness } \\
\left\langle s_{i} t_{j}\right\rangle \leqq\left\langle s_{i}\right\rangle\left\langle t_{j}\right\rangle, \\
\left\langle\sigma_{m} t_{j}\right\rangle \leqq\left\langle\sigma_{m}\right\rangle\left\langle t_{j}\right\rangle, \\
\left\langle s_{i} s_{j}\right\rangle \geqq\left\langle s_{i}\right\rangle\left\langle s_{j}\right\rangle, \\
\left\langle s_{i} \sigma_{m}\right\rangle \geqq\left\langle s_{i}\right\rangle\left\langle\sigma_{m}\right\rangle, \\
\left\langle t_{i} t_{j}\right\rangle \geqq\left\langle t_{i}\right\rangle\left\langle t_{j}\right\rangle .
\end{gathered}
$$

Obviously $(7 \mathrm{~b})$ and $(7 \mathrm{~d})$ apply to the $(2 N+1)$-layer case only.

Equations $(7 \mathrm{a})-(7 \mathrm{c})$ imply that an increase of the field on site $i$, together with a decrease of the field on site $-i$ by the same amount, lowers the expectation value of $s_{j}$ or $\sigma_{m}$ and raises the expectation value of $t_{j}$. A consequence is that in the $(2 N+1)$-layer system with + boundary above and - boundary below, the magnetization of the central layer increases as a function of $N$, whereas in the $2 N$-layer system the difference between the magnetizations of the layers 1 and -1 , and hence the magnetization of the layer 1 itself, decreases with $N^{2}$.

Furthermore it is possible to say something about the behaviour of $\left\langle\sigma^{(n)}\right\rangle$, which will denote the average magnetization per site in the layer $n$. To this end consider the $2 N$-layer system. We start with a situation in which in $(6 \mathrm{~b}) h_{i}=h>0$ if $i$ is a boundary site, $h_{i}=0$ otherwise and $H_{i}=0$ for all $i$. Next we change $H_{i}$ to $2 h$ for the boundary spins on the layer 1 , which means that we create a situation in which the boundary fields on the layers $-1,2,3 \ldots$ are $h$, those on the layer 1 are $3 h$ and those on the layers $-2,-3 \ldots$ are $-h$. As a consequence of $(6 a)$ the expectation value of none of the $t$ variables is increased by this change. The expectation value of a $t$ variable in layer 1 is $\frac{1}{2}\left\langle\sigma^{(1)}-\sigma^{(-1)}\right\rangle$ on the average. With the new boundary conditions however this corresponds to $\frac{1}{2}\left\langle\sigma^{(2)}-\sigma^{(1)}\right\rangle$ in the original situation, provided one takes the limits of $h$ and $N$ going to $\infty$. By next increasing also the fields $H_{i}$ on the boundary sites of the layers $2,3, \ldots$ the expectation value of all $t$ variables is lowered again. Therefore we may conclude that in the limit of $h$ and $N$ going to $\infty$ $\left\langle\sigma^{(n+1)}-\sigma^{(n)}\right\rangle \leqq\left\langle\sigma^{(n)}-\sigma^{(n-1)}\right\rangle$ for all $n>1$; in other words $\left\langle\sigma^{(n)}\right\rangle$ is a concave function of $n$ for $n>0$.

\section{Concluding Remarks}

By the simple method sketched in Section 2 we have obtained a lower bound for the roughening temperature $T_{R}$ of an interface in the three-dimensional Ising model, which is much higher than the lower

\footnotetext{
${ }^{2}$ Lebowitz has pointed out that (6a) can be used to prove our main result in an alternative way: By setting all the $h_{i}$ (but not the $H_{m}$ ) equal to $+\infty$ the central layer becomes equivalent to a two-dimensional Ising system. By subsequently relaxing the $h_{i}$ values the magnetization of the central layer is not decreased.
} 
bound obtained by completely different methods by Dobrushin. On the other hand Dobrushin obtains several additional results which are not reproduced by our method.

Application of our method to the phase separation line in a twodimensional Ising system yields no information; in that case it comes out that the magnetization of a central row is not lower than the magnetization of a one-dimensional Ising chain, which is zero at each nonzero temperature.

It would be desirable also to have a non-trivial upper bound for the temperature $T_{R}$. If one could find an upper bound below the critical temperature, this would prove the possibility of more than one phase transition in the three-dimensional Ising model. However, not even for the solid-on-solid model, where the critical temperature is infinite, such an upper bound for $T_{R}$ has been found. On the other hand the estimates of $T_{R}$ provided for this model by computer experiments [7] are of the same order of magnitude as for the isotropic model.

Acknowledgements. I want to thank G. Gallavotti and J. L. Lebowitz for stimulating discussions and valuable criticism.

\section{References}

1. Dobrushin, R.L.: Theory Probability Appl. 17, 582 (1972)

2. Gallavotti, G.: Commun. math. Phys. 27, 103 (1972)

3. Weeks, J.D., Gilmer, G. H., Leamy, H. J.: Phys. Rev. Letters 31, 549 (1973)

4. Lebowitz, J. L.: Commun. math. Phys. 35, 87 (1974)

5. Griffiths, R.B.: Math. Phys. 8, 478, 484 (1967); Kelly,D. G., Sherman, S.: J. Math. Phys. 9, 466 (1968)

6. Fortuin, C.M., Kasteleyn,P.W., Ginibre, J.: Commun. math. Phys. 22, 89 (1971)

7. Gilmer, G.H., Bennema,P.: J. Crystal Growth 13/14, 148 (1972); Leamy, H.J., Gilmer, G. H. : Preprint 